\title{
Europa treibt die IT-Sicherheit voran
}

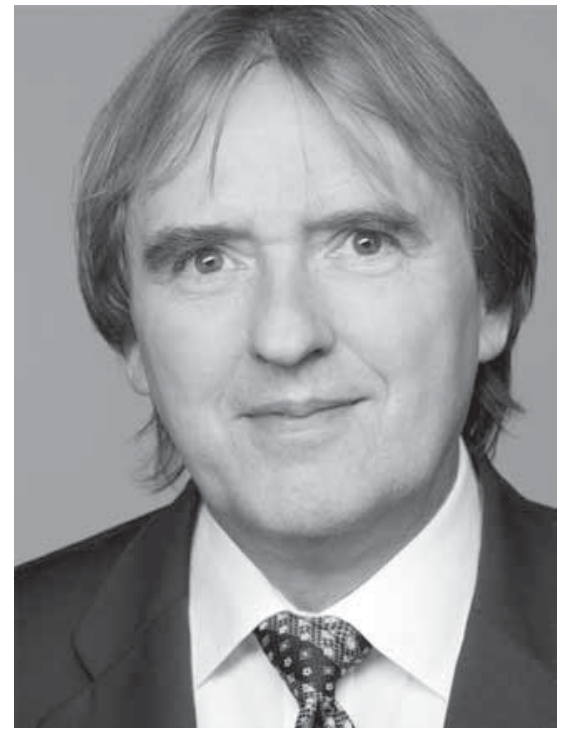

Europa hat in den letzten Jahren sehr viel Energie in die Gesetzgebungen im Bereich der IT-Sicherheit und des Datenschutzes investiert.

Zum einen die Datenschutz-Grundverordnung (DSGVO), eine Verordnung, mit der die Regeln zur Verarbeitung personenbezogener Daten durch private Unternehmen und öffentliche Stellen EU-weit vereinheitlicht werden. Dadurch soll einerseits der Schutz personenbezogener Daten innerhalb der Europäischen Union sichergestellt, andererseits der freie Datenverkehr innerhalb des Europäischen Binnenmarktes gewährleistet werden.

Dann eIDAS (electronic Identification, Authentication and trust Services), eine Verordnung über elektronische Identifizierung und Vertrauensdienste für elektronische Transaktionen im EU-Binnenmarkt, die noch mehr einheitliche Vertrauensdienste in Europa motivieren soll.

Zusätzlich wurde die NIS-Richtlinie erlassen, die als europäisches Rahmenwerk das Ziel verfolgt, in allen Mitgliedstaaten der Europäischen Union ein hohes gemeinsames Sicherheitsniveau von Netz - und Informationssystemen zu erreichen, damit wirtschaftliche und gesellschaftliche Tätigkeiten und insbesondere der Binnenmarkt reibungslos funktionieren können.

Außerdem wird an der e-Privacy-Verordnung sowie an einer Verordnung über einen Rahmen für den freien Verkehr nicht personenbezogener Daten in der Europäischen Union gearbeitet und weitere Initiativen sollen folgen.

Zu diesem regulatorischen Themenfeld haben sich in dieser DuD-Ausgabe verschiedene Fachleute mit unterschiedlichen Sichtweisen - auch mit kritischen Stellungnahmen - geäußert, um uns einen tieferen Einblick in die verschiedenen Gesetze zu ermöglichen.

Europa will in allen Bereichen enger zusammenwachsen. Cyber-Sicherheit ist dabei ein bedeutender Faktor. Die Kommission hat dazu alle Firmen und Organisationen der Mitgliedstaaten aufgerufen, zusammen das Thema Cyber-Sicherheit zu schärfen und voran zu bringen. Der Artikel "Cybersecurity made in EU“ soll hier einen Überblick schaffen.

Zwei weitere Beiträge dieses Heftes widmen sich der Frage, wie IT-Sicherheit und Datenschutz durch Maschinelles Lernen und mit Hilfe Künstlicher Intelligenz weiterentwickelt werden können. Erläutert werden die Grundlagen und Grenzen sowie die Anwendungsperspektiven dieser Konzepte.

Der Artikel "Was die IT-Sicherheitsindustrie und Anwender voneinander lernen können" zeigt die Herausforderungen, die wir noch gemeinsam lösen müssen.

Wir wünschen Ihnen viel Freude und interessante Erkenntnisse beim Studium der Beiträge dieser DuD-Ausgabe. Auch über Ihr Feedback freuen wir uns sehr.

\section{Norbert Pohlmann}

